ANNALES

POLONICI MATHEMATICI

$85.3(2005)$

\title{
Connectedness of the Carathéodory discs for doubly connected domains
}

\author{
by LEONHARD FreRick (Wuppertal) and \\ Gerald Schmieder (Oldenburg)
}

\begin{abstract}
We prove that the Carathéodory discs for doubly connected domains in the complex plane are connected.

Let $G \varsubsetneqq \overline{\mathbb{C}}$ be a domain and assume that no boundary component is a point. The Carathéodory distance $c\left(z_{0}, z_{1}\right)$ between two points $z_{0}, z_{1} \in G$ is defined as $\sup \left|f\left(z_{0}\right)\right|$, where the supremum is taken over all $f$ holomorphic in $G$ whose modulus is bounded by 1 and which vanish at $z_{1}$. The following theorem proves a conjecture of Pflug and Jarnicki ([1, p. 42]) in the doubly connected case:
\end{abstract}

THEOREM 1. If the boundary of $G$ consists of two continua and $z_{0} \in G$, then the Carathéodory discs $\left\{z \in G: c\left(z, z_{0}\right)<r\right\}, r \in(0,1)$, are connected.

Proof. It is enough to show that for an arbitrary $z_{1} \in G$ there is a curve connecting $z_{0}$ and $z_{1}$ on which $c\left(\cdot, z_{0}\right)$ is increasing. Since $c$ is invariant under conformal maps, we may replace $G$ by its image under the Möbius transformation $z \mapsto\left(z-z_{0}\right) /\left(z-z_{1}\right)$, and therefore we may assume that $z_{0}=0, z_{1}=\infty$. Moreover there is some conformal mapping onto some radial slit domain which leaves 0 and $\infty$ fixed (see e.g. [2, Theorem IX.24]). Since rotations also fix 0 and $\infty$ we can arrange that $\partial G$ is contained in $\{z \in \mathbb{C}: \Re(z) \leq 0\}$.

Using the continuity of $c$ it is enough to show that $c(\cdot, 0):(0, \infty) \rightarrow(0,1)$, $t \mapsto c(t, 0)$, is increasing. Let therefore $t_{1}>t_{0}>0$ be arbitrary. Note that $\varphi(z)=\left(z-t_{1}\right) /\left(z-t_{0}\right)$ maps the closed left halfplane onto the closed disc bounded by the circle through $1=\varphi(\infty)$ and $t_{1} / t_{0}=\varphi(0)>1$, which is symmetrical about the real axis. This shows that $1<|\varphi(z)|<t_{1} / t_{0}$ for each

2000 Mathematics Subject Classification: 30C75, 30C80.

Key words and phrases: Carathéodory distance. 
$z \in \partial G$ and $m=\max \{|\varphi(z)|: z \in \partial G\}<\varphi(0)$. We set $h(z):=\varphi(z) / m$ and obtain $h(0)>1$.

Let $f$ be the unique holomorphic function in $G$, whith modulus bounded by 1 , which vanishes at $t_{0}$ and fulfills $c\left(t_{0}, 0\right)=c\left(0, t_{0}\right)=f(0)$. If $g:=f h$, then $g$ is holomorphic on $G$, its modulus is bounded by 1 and it vanishes at $t_{1}$. Therefore $c\left(t_{0}, 0\right)=f(0)<g(0) \leq c\left(0, t_{1}\right)=c\left(t_{1}, 0\right)$ and the proof is complete.

\section{References}

[1] M. Jarnicki and P. Pflug, Invariant Distances and Metrics in Complex Analysis, de Gruyter, Berlin, 1993.

[2] M. Tsuji, Potential Theory in Modern Function Theory, Chelsea, New York, 1975.

Fachbereich 7 - Mathematik

Bergische Universität - Gesamthochschule Wuppertal

Gaußstraße 20

42119 Wuppertal, FRG

E-mail: Leonhard.Frerick@math.uni-wuppertal.de

Fakultät V

Institut für Mathematik

Universität Oldenburg

26111 Oldenburg, FRG

E-mail: schmieder@mathematik.uni-oldenburg.de 